\title{
Especificação e desenvolvimento de um ambiente educativo móvel para a prática da escrita Braille
}

\author{
Antonio Rodrigo S. Silva ${ }^{1}$, Agebson Rocha Façanha ${ }^{1}$, Windson Viana ${ }^{2}$, José Aires \\ de Castro Filho ${ }^{3}$, Jaime Sánchez ${ }^{4}$ \\ ${ }^{1}$ Instituto Federal de Educação, Ciência e Tecnologia do Ceará - Fortaleza - CE - Brasil \\ ${ }^{2}$ Mestrado e Doutorado em Ciências da Computação - Universidade Federal do Ceará - \\ Fortaleza - CE - Brasil \\ ${ }^{3}$ Instituto UFC Virtual - Universidade Federal do Ceará - Fortaleza - CE - Brasil \\ ${ }^{4}$ Departamento de Ciencias de la Computación y Centro de Investigación Avanzada en \\ Educación - Universidad de Chile - Santiago - Chile \\ rodrigosantoseifce.edu.br, agebsoneifce.edu.br, \\ windson@virtual.ufc.br, aires@virtual.ufc.br, jsanchez@dcc.uchile.cl
}

\begin{abstract}
Resumo.O presente trabalho apresenta os primeiros esforços do desenvolvimento do GBraille Suite, que é composto por softwares educativos acessiveis focados na aprendizagem e na prática do Português através da escrita Braille. Esse artigo apresenta o design, desenvolvimento e avaliação inicial de um teclado virtual baseado na metáfora Braille e, a partir dele, foi desenvolvido um aplicativo acessivel para a prática de ortografia e sinonímia que utiliza interfaces multimodais (gestos na tela, voz e vibrações). A avaliação de usabilidade inicial realizada com alunos com deficiência visual do ensino fundamental indica uma boa aceitação dos aplicativos.
\end{abstract}

\begin{abstract}
This paper presents our first efforts to develop GBraille Suite, which consists of accessible educational software focused on learning and practicing of Portuguese through the Braille writing system. This paper presents the design, the development and the initial evaluation of a virtual keyboard based on Braille metaphor and, from this keyboard, it was developed a mobile application to practice spelling and synonymy using multimodal interfaces (screen gestures, voice and device vibration). The initial usability evaluation conducted with visually impaired students of elementary school indicates good acceptance of the applications.
\end{abstract}

\section{Introdução}

Dispositivos móveis, como smartphones e tablets, se tornaram dispositivos de uso cotidiano e verdadeiros centros de informação e entretenimento [VIANA et al. 2011]. Tais dispositivos oferecem às pessoas acesso generalizado a uma grande gama de aplicações: serviços de SMS / MMS, clientes de redes sociais, e-books, jogos e navegação na Web. Esses dispositivos também já se mostraram efetivos no auxílio aos processos de ensino e aprendizagem em diversas áreas do conhecimento (e.g., medicina, português, biologia e matemática) para melhoria de processos cognitivos tanto em crianças quanto em adultos [PEREIRA; RODRIGUES 2013]. Como tecnologia acessível, os dispositivos móveis também se mostram importantes aliados em função da 
diversidade de recursos que podem auxiliar as pessoas com deficiência visual em suas atividades cotidianas. O acesso a esses recursos é proporcionado pelas interfaces multimodais desses dispositivos que se baseiam no uso de feedback táctil e sonoros combinados com gestos nas telas do dispositivo [FAÇANHA et al. 2014].

Este trabalho de pesquisa é focado na inserção das tecnologias móveis para o auxílio à aprendizagem e a prática da escrita Braille. O Braille é um sistema de leitura e escrita tátil mais utilizado por pessoas com deficiência visual. É por meio dele que os alunos deficientes visuais tomam contato com a grafia, permitindo a compreensão de letras, números, sinais de pontuação e símbolos. A escrita Braille tem forte relação com a aprendizagem do Português, pois é através dela que a pessoa com deficiência visual entra em contato com a estrutura dos textos, a ortografia das palavras e a pontuação. É, ainda, o único meio de leitura disponível para os surdos-cegos.

Apesar da importância da escrita Braille em sala de aula, o uso dos instrumentos tradicionais apresentam algumas dificuldades. Conforme relata Kalra et al. [2007], a reglete e o punção exigem que o aluno escreva da direita para a esquerda (ao contrário da forma como ele lê) e não há um feedback imediato das palavras escritas, pois é necessário virar a folha para ler. As máquinas Perkins, uma alternativa para escrita Braille, tem um alto custo que impede que as escolas adquiram esses equipamentos em maior quantidade. Com tais dificuldades, os alunos podem desenvolver uma insatisfação na realização de produções textuais em que necessitem usar essa simbologia.

Recentemente, surgiu uma gama de soluções para entrada de texto em dispositivos móveis que propõem teclados físicos e virtuais que usam a metáfora do Braille como, por exemplo, o Swipe Type [PEBAM 2011], Braille Type [OLIVEIRA et al. 2011], BrailleTouch [SOUTHERN et al. 2012], Perkinput [AZENKOT et al. 2012], Upsense Keyboard [INPRIS 2012] e LêBraille [FAÇANHA et al. 2014]. Esses projetos propõem teclados virtuais que exibem uma célula Braille em uma tela sensível ao toque. Cada um deles possui uma estratégia própria de inserção de um símbolo Braille, alternando entre os gestos aplicados sobre a tela, o suporte a toques únicos ou simultâneos e o uso de um ou vários dispositivos.

Essa pesquisa, portanto, combina um teclado virtual baseado na metáfora Braille com práticas lúdicas em dispositivos móveis. O objetivo é criar uma gama de aplicativos móveis que possam ser utilizados, em sala de aula ou não, para estimular a escrita Braille e o exercício da escrita em Português. Neste artigo, apresentamos o design, desenvolvimento e avaliação do GBraille Suite. Esse aplicativo móvel é composto uma interface Launcher ("Menu Inicial" do sistema operacional ) acessível, um teclado virtual baseado na metáfora Braille e um aplicativo multimodal lúdico para o exercício de ortografia e sinonímia, o GBraille Hangman.

Este artigo está organizado em seis seções: 1. Introdução; 2. GBraille Suite. 3. Experimentação inicial; 4. Trabalhos relacionados; 5. Considerações finais e trabalhos futuros; e 6. Agradecimentos.

\section{GBraille Suite}

A suíte de aplicativos GBraille foi desenvolvida para a plataforma Android e baseia-se em interfaces multimodais, nas quais o uso de um sintetizador de voz é o elemento principal para promover a acessibilidade. As interfaces dos aplicativos seguem as recomendações para acessibilidade em dispositivos móveis sugeridas em [FAÇANHA 
et al. 2014], que foram propostas a partir de testes de aceitação com pessoas com deficiência visual.

O GBraille Suite é composto pelos seguintes aplicativos: a) GBraille Launcher, uma alternativa de interface acessível que será exibida ao aluno com deficiência visual no momento em que ele ligar o telefone. b) GBraille Keyboard, uma solução de entrada de dados em Braille para dispositivos móveis; c) O GBraille Hangman, um aplicativo lúdico educativo no qual os alunos precisam adivinhar uma palavra, com base em uma informação que é repassada pelo software. A Figura 1 ilustra capturas de telas dos aplicativos. É importante ressaltar que alguns elementos visuais estão presentes nessas interfaces, pois podem contribuir na compreensão dos elementos da aplicação no caso de usuários com baixa visão.

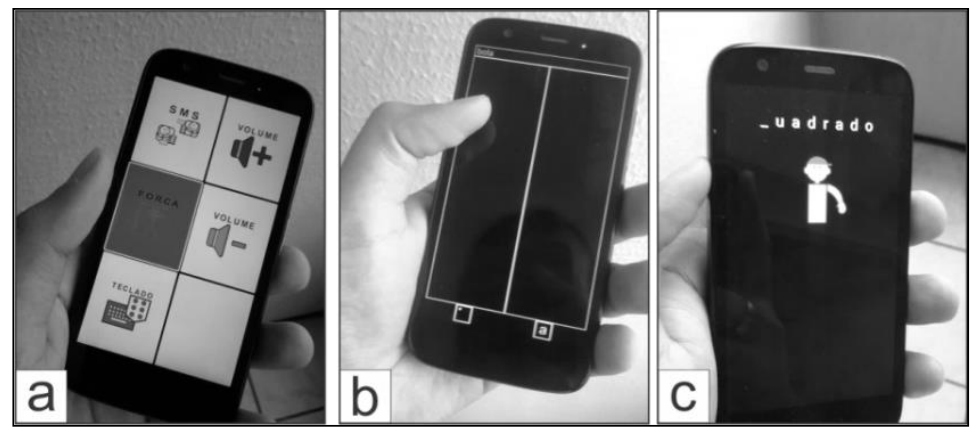

Figura 1 - a) GBraille Launcher; b) GBraille Keyboard; c) GBraille Hangman

\subsection{GBraille Launcher}

Uma das dificuldades no uso de aplicativos móveis acessíveis em dispositivos com telas de toque tem relação com o menu inicial dos sistemas operacionais. Esses menus tem forte apelo visual e, mesmo com aplicativos de auxílio à navegação (e.g., VoiceOver), podem dificultar o uso por parte de usuários com deficiência visual. Nesse contexto, o GBraille Launcher é uma interface inicial que dispõe os aplicativos e opções em duas colunas e três linhas, disposição semelhante à assumida pelos pontos da célula Braille. Quando um aplicativo é selecionado, há a emissão de um sinal sonoro e a síntese de voz é ativada, descrevendo o ícone. Uma configuração do aplicativo faz com que o mesmo seja ativado sempre que o dispositivo é inicializado.

Outros recursos que promovem a acessibilidade do GBraille Launcher são: a regulagem do volume do áudio, ajuste de contraste e ampliação de tela. São considerados diferenciais: (i) a possibilidade de personalização, tanto dos aplicativos disponíveis ao aluno quanto nos gráficos de cada opção; (ii) suporte a áudio estéreo, dividindo o som no lado esquerdo ou direito nos fontes de ouvido, permitindo uma melhor orientação do aluno na área de toque; (iii) identificação sonora do menu em utilização quando o usuário balança o celular (movimento conhecido como shake).

\subsection{GBraille Keyboard}

A base do sistema Braille é uma célula retangular formada pela combinação de seis pontos em relevo, dispostos em três linhas e duas colunas. Esta disposição permite a formação de 64 combinações [REYNOLDS; VANNEST; FLETCHER-JANZEN 2013]. O GBraille Keyboard permite a inserção de todos esses símbolos com apenas três gestos na tela. Um projeto anterior foi desenvolvido um teclado visual baseado em Braille, o LêBraille [FAÇANHA et al. 2014]. Entretanto, apesar de permitir a entrada de dados 
com essa metáfora, os tempos de escrita eram bastante impeditivos para seu uso como instrumento de aprendizagem ou prática do Braille.

O GBraille Keyboard foi, então, desenvolvido com base em outra abordagem, o TypeInBraille [Oliveira 2011]. Sua forma de funcionamento permite que um símbolo Braille seja inserido em três passos e com múltiplos toques, deixando mais rápido o processo de escrita. Inicia-se escolhendo um dos pontos localizados no topo da célula Braille (pontos 1 e 4), prosseguindo com os pontos do meio (pontos 2 e 5) e finalizando com os pontos da base (pontos 3 e 6). Em cada um desses passos é possível realizar um dos seguintes movimentos ilustrados na Figura 2. Um vídeo da execução do teclado encontra-se disponível em: http://goo.gl/DcMxG6.

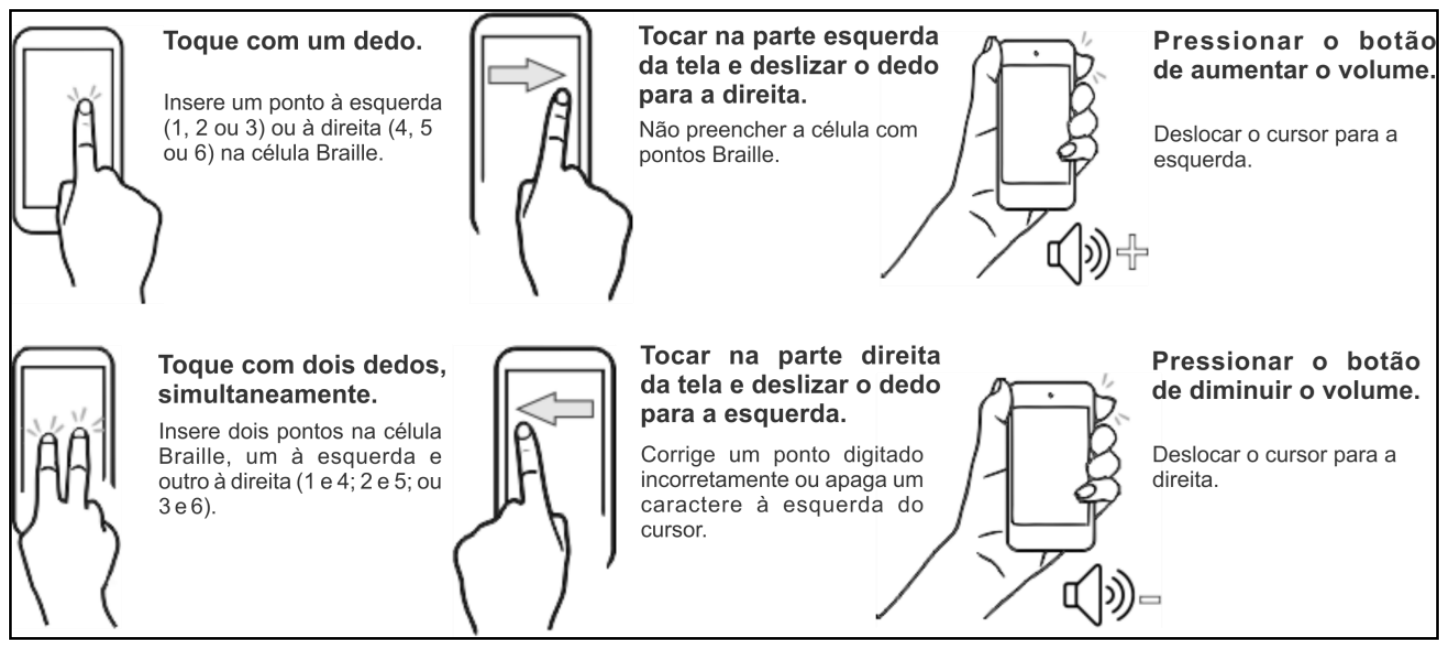

Figura 2 - Gestos para inserção de caracteres no GBraille Keyboard

Um das características particulares do GBraille Keyboard está na forma como ele responde aos toques na tela. Cada ponto teve um som associado de acordo com a altura do som. Os pontos da primeira linha (1 e 4) são agudos, o da segunda linha (2 e 5) são intermediários e o da terceira linha ( 3 e 6) são graves. Assim os toques podem ser distinguidos pelo usuário mais facilmente (Figura 3). O teclado também proporciona: (i) rapidez na digitação de um caractere; (ii) percepção espacial dos pontos digitados por meio da saída no canal esquerdo ou direito de um fone de ouvido; (iii) suporte à vários sintetizadores de voz instalados no dispositivo; (iv) integração a outras aplicações.

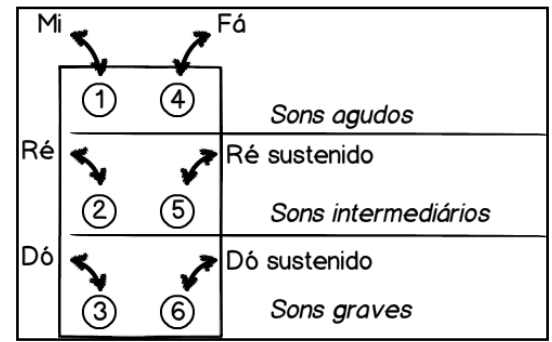

Figura 3 - Associação da linha/coluna de inserção e o som emitido

\subsection{GBraille Hangman}

O GBraille Hangman é um jogo do tipo perguntas e respostas, baseado no tradicional modelo do Jogo da Forca. Para cada jogada é exibida uma pergunta, em que o aluno deve encontrar uma resposta por meio do preenchimento das letras de uma palavra. $\mathrm{O}$ jogador possui seis tentativas para acertar a palavra por completo. Para cada tentativa, o 
software oferece um feedback por meio de síntese de voz, informando de forma clara o progresso obtido. Para o design do jogo, foram realizadas entrevistas com professores de educação especial e uma análise de jogos similares existentes no DOSVOX.

O jogo integra-se facilmente aos currículos escolares por permitir a personalização das atividades que serão disponibilizadas aos alunos, auxiliando-os na aquisição de conhecimentos em diversos tópicos da Língua Portuguesa. Os docentes podem cadastrar suas próprias perguntas e respostas, e atribuir a cada uma delas diferentes níveis de dificuldade. Na versão atual estão presentes os níveis Fácil, em que o aluno deve acertar uma única letra da palavra, e Difícil, que requer o preenchimento de todas as letras. Por meio desta estratégia, o professor de Língua Portuguesa pode trabalhar diversos assuntos, conforme ilustra a tabela a seguir.

Tabela 1 - Assuntos trabalhados no GBraille Hangman

\begin{tabular}{|l|l|}
\hline Nível Fácil & Ortografia; paronímia. \\
\hline Nível Difícil & Sinonímia e antononímia; substantivos coletivos; plural de palavras. \\
\hline
\end{tabular}

Além da dificuldade, o aluno é estimulado por meio dos elementos de motivação, como a aleatoriedade de exibição de perguntas e a regulação do tempo de cada jogada por meio de um cronômetro: caso o aluno resolva a atividade em um tempo inferior ao informado pelo professor, uma bonificação é atribuída. Um ranking conterá a pontuação e o registro de vitórias consecutivas em cada um dos níveis. Na presença do erro, situação comum e inevitável no cotidiano escolar, é disponibilizada ao aluno a opção "Ajuda", que dispõe de uma explicação sobre a atividade que está em prática. Assim, os educandos podem avaliar o resultado de suas ações e refletir sobre as correções possíveis a serem realizadas, propiciando dessa forma a autonomia pela busca do conhecimento por meio do erro construtivo. O link a seguir exibe um vídeo com a execução do aplicativo feita por um aluno do Ensino Fundamental que possui deficiência visual: http://goo.gl/Ajz6FB.

\section{Experimentação inicial}

\subsection{Ferramentas}

Para a experimentação foram utilizados, inicialmente, protótipos táteis de baixa fidelidade, feitos em isopor e com texturas em alto relevo. Em seguida os softwares foram apresentados aos usuários em um smartphone Samsung Galaxy S4, rodando a versão 4.2 do Android e utilizando o sintetizador de voz Loquendo TTS. Optou-se pela utilização desta plataforma pela sua facilidade de desenvolvimento e por ser o sistema predominante em $81.1 \%$ dos smartphones [IDC 2014]. O dispositivo foi escolhido por apresentar as características mais apropriadas para o projeto: tamanho, peso, resolução de tela, capacidade de disco e facilidade na configuração e instalação dos softwares.

\subsection{Instrumentos}

Três questionários foram elaborados para aplicação junto aos alunos (Figura 4). Cada questionário correspondia a um software (Launcher, Keyboard e Hangman) e foi criado com bases nos trabalhos de SAVI et al. (2010), que propôs um modelo de questionário baseado nos modelos ACRS de John Keller, Kirkpatrick e na taxonomia de Bloom. Para mensurar o grau de concordância dos sujeitos quanto às declarações apresentadas, utilizou-se uma escala do tipo Likert de cinco pontos, sendo $1=$ discordo totalmente e 5 = concordo totalmente. Ainda segundo o modelo, cada item pode ser avaliado em três 
aspectos, cada um deles contendo atributos, a saber: motivação (atenção, relevância, confiança e satisfação), experiência do usuário (imersão, desafio, habilidade, competência, divertimento, controle e interação social) e impacto na aprendizagem (conhecimento, compreensão e aplicação).

\begin{tabular}{|c|c|c|}
\hline Q1 - Gbraille Launcher & Q2 - Gbraille Keyboard & Q3 - Gbraille Hangman \\
\hline $\begin{array}{l}\text { DL1:Consigo navegar sem dificuldades }(\mathrm{C}) \\
\text { DL2:Compreendo os textos narrados na tela } \\
\text { (H) } \\
\text { DL3:Consigo me localizar rapidamente na } \\
\text { tela(H) } \\
\text { DL4: Consigo abrir os aplicativos } \\
\text { facilmente }(\mathrm{H}) \\
\text { DL5:Percebo que o design do aplicativo } \\
\text { remete à ideia de uma célula Braille }(\mathrm{R}) \\
\text { DL6: Me senti bem durante a navegação }(\mathrm{S})\end{array}$ & \multirow{2}{*}{$\begin{array}{l}\text { DK1:As ações do teclado são de fácil } \\
\text { compreensão (C) } \\
\text { DK2:O teclado é de fácil utilização (C) } \\
\text { DK3: Com o te clado posso digitar } \\
\text { rapidamente(S) } \\
\text { DK4:Me senti bem durante a digitação (S) } \\
\text { DK5:O teclado prende minha atenção (A) } \\
\text { DK6:Consigo relacionar o teclado com uma } \\
\text { célula Braille (R) } \\
\text { DK7:Acredito que poderia utilizar o teclado } \\
\text { para interagir com outros softwares para a } \\
\text { minha aprendizagem (R) } \\
\text { DK8:Senti que estava tendo progresso com } \\
\text { o uso do teclado (H) }\end{array}$} & $\begin{array}{l}\text { D H 1: As perguntas são de fácil } \\
\text { compreensão(C) } \\
\text { DH2: As respostas foram fáceis de serem } \\
\text { digitadas (C) } \\
\text { DH3: Gostei do assunto tratado no jogo } \\
\text { (R) } \\
\text { DH4:Consigo reconhecer a grafia correta } \\
\text { das palavras apresentadas no jogo(H) } \\
\text { DH5:Ojogo édivertido(D) } \\
\text { DH6:Me senti bem durante a utilização do }\end{array}$ \\
\hline $\begin{array}{l}\text { A - Atenção } \\
\text { C - Confiança } \\
\text { D- Diversão } \\
\text { H - Habilidade } \\
\text { R - Relevância } \\
\text { S - Satisfação }\end{array}$ & & $\begin{array}{l}\text { jogo(S) } \\
\text { DH7:O jogo me manteve mot } \\
\text { continuarutilizando-o(D) } \\
\text { DH8:Alcancei os objetivos do jogo } \\
\text { DH9:Eu jogaria este jogo novament }\end{array}$ \\
\hline
\end{tabular}

Figura 4 - Questionários

\subsection{Usuários}

Para a avaliação do GBraille Keyboard foram convidados três pessoas que participaram da avaliação proposta por Façanha [2014] que consistia na análise de desempenho teclados que usam a metáfora do Braille e outros com o layout tradicional QWERTY. Já para a avaliação do GBraille Launcher e GBraille Hangman foram convidados dois alunos de Ensino Fundamental de uma escola para crianças, adolescentes e adultos com deficiência visual. O perfil dos participantes encontra-se detalhado na Tabela 2.

Tabela 2 - Perfil dos usuários

\begin{tabular}{|l|l|l|l|l|l|}
\hline & \multicolumn{1}{|c|}{ usuário 1 } & \multicolumn{1}{|c|}{ usuário 2 } & \multicolumn{1}{c|}{ usuário 3 } & \multicolumn{1}{c|}{ aluno 1 } & \multicolumn{1}{c|}{ aluno 2 } \\
\hline Idade & 29 & 27 & 35 & 20 & 17 \\
\hline Gênero & Masculino & Masculino & Masculino & Feminino & Masculino \\
\hline Perfil & Cego & Cego & Cego & Cego & Cego \\
\hline Domínio do Braille & Intermediário & Avançado & Intermediário & Intermediário & Intermediário \\
\hline
\end{tabular}

\subsection{Procedimento}

A primeira etapa consistiu em observações sobre o uso GBraille Keyboard com o objetivo de averiguar a rapidez e a satisfação de uso do teclado proposto, bem como verificar possíveis problemas de acessibilidade e de usabilidade. Participaram deste momento os usuários 1, 2 e 3, que receberam orientações iniciais sobre o layout do teclado, sobre a metodologia de inserção de dados no teclado virtual. Após um momento de exploração livre, os usuários foram convidados a escrever um dito popular ${ }^{1}$ para comparar a velocidade de digitação entre os teclados GBraille e LêBraille.

A segunda etapa compreendeu a realização de uma atividade com os alunos $1 \mathrm{e}$ 2. Foram realizados três encontros, onde foram apresentados o GBraille Launcher, o GBraille Keyboard e o GBraille Hangman, nesta ordem. Para o GBraille Launcher

\footnotetext{
${ }^{1}$ Antes de dar comida a um mendigo, dá-lhe uma vara e o ensina a pescar. Esta frase também foi inserida pelos participantes durante a avaliação do teclado LêBraille.
} 
houve uma explicação básica e os alunos foram solicitados a realizar as seguintes atividades: (i) diminuir e aumentar o volume ao máximo que puder; (ii) Acessar os aplicativos presentes na interface. $O$ tempo total desta atividade foi de 60 minutos.

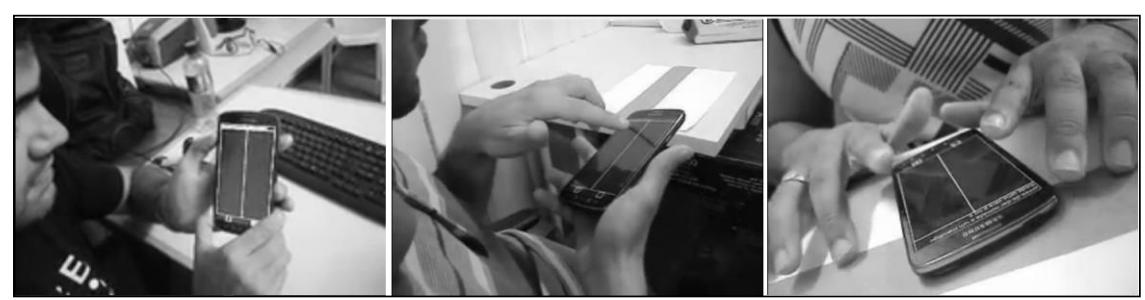

Figura 5 - Validação do teclado GBraille

Para o GBraille Keyboard foi disponibilizado um encontro de 90 minutos, onde o pesquisador inicialmente apresentou os gestos básicos, fez exemplificações da escrita do alfabeto e procedeu com um tempo para livre uso do teclado. Para o GBraille Hangman foi feita uma revisão rápida sobre o funcionamento do teclado, seguida da conceituação básica do jogo. Por fim o aluno foi convidado a responder a, no mínimo, cinco perguntas no jogo. O tempo total desta atividade foi de 60 minutos.

\subsection{Resultado}

\subsection{1 - Resultados da avaliação da velocidade de escrita}

O Gráfico 1 mostra os resultados obtidos na primeira etapa, que avaliam o tempo de escrita do dito popular em cinco modalidades. O tempo de quatro teclados (Braille, QWERY Físico, QWERTY VIRTUAL CPQD e LêBraille) foram obtidos do trabalho de Façanha [2014]. Os dados apontam para um tempo de escrita no GBraille Keyboard bem menor do que no uso teclado LêBraille, embora, ainda bem acima do tempo do uso do Braille tradicional. Apesar deste fato, o teclado já possui desempenho aceitável para práticas textuais em sala de aula (chats, debates, perguntas e respostas, escrita colaborativa) em que a rapidez é fundamental no sucesso da realização das atividades em função do tempo limitante da aula. Vale ressaltar ainda, que esses tempos foram medidos na primeira interação dos usuários com o teclado e, pressupõe-se que com o uso continuado, os tempos de escrita tendem a diminuir.

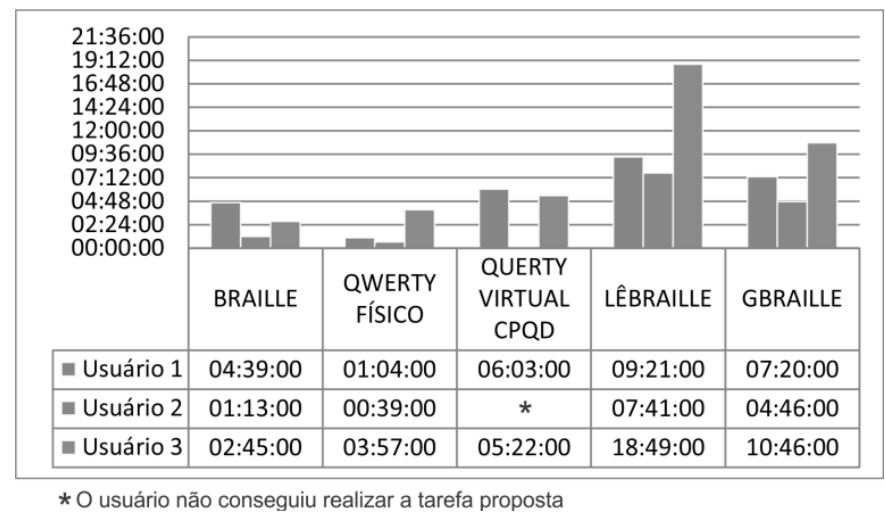

Gráfico 1 - Tempo de escrita do dito popular

\subsection{2 - Resultados da avaliação da velocidade de escrita}

Para a segunda etapa, procedeu-se com a análise dos questionários preenchidos pelos alunos, onde se constatou que: 
1 - A interface inicial do GBraille agradou aos usuários (DL6) e que o seu uso torna-se facilitado graças aos recursos de acessibilidade disponibilizados (DL1, DL3 e DL4). Os usuários conseguiram compreender a disposição dos elementos na tela e o que era descrito por meio da síntese de voz (DL2 e DL5).

2 - Os alunos estavam satisfeitos com o teclado (DK4) e com a forma diferenciada de inserção de caracteres, que prendeu a atenção dos mesmos (DK5). Apesar de declararem que conseguiam fazer a associação entre teclado célula Braille (DK6), alguns apresentaram mais facilidade em utilizá-lo e outros, um pouco mais de dificuldade (DK1, DK2 e DK3). O teclado era utilizado com mais eficiência com o decorrer do tempo (DK8) e eles gostariam de utilizá-lo novamente em outros softwares (DK7).

3 - O Jogo da Forca foi considerado acessível (DH1), agradável (DH6) e divertido (DH5, DH7 e DH9), e que todos gostaram da forma como ele foi inserido no contexto educativo (DH3). Contudo um aluno alegou dificuldades quanto à inserção das respostas (DH2, DH4 e DH8), pois alegou que houve uma grande distância de tempo entre a prática inicial do teclado e a utilização do jogo e que o mesmo foi feito em um curto espaço de tempo.

\section{Trabalhos Relacionados}

São encontradas na literatura algumas iniciativas de auxílio ao ensino e a prática do Braille de forma lúdica, em diversas plataformas como computador e dispositivos móveis. Por exemplo, o Braille Virtual ${ }^{2}$ e o eKodBraille ${ }^{3}$ contem recursos visuais e animações e estão destinados aos videntes ${ }^{4}$, estando inacessíveis às pessoas com deficiência visual. Com enfoque na educação especial, se destacam o TiMproject [ARCHAMBAULT; BURGER 2000] que objetiva o desenvolvimento de jogos educacionais para crianças cegas usando um equipamento táctil (Multimodal) ligado ao computador. Já o Body-Braille [OHTSUKA et al. 2008] e o UbiBraille [NICOLAU et al. 2013] propiciam um retorno dos pontos em Braille através de vibração de sensores dispostos sobre o corpo do usuário. É possível, inclusive, desenvolver e embarcar alguns jogos no hardware do Body-Braille. Assim, a interação com os diversos dispositivos, se dá através de teclas recobertas (opcionalmente) com pontos em Braille, áudio ou mesmo vibração, visando orientar o posicionamento dos dedos do usuário e a digitação com a Máquina Perkins, além de facilitar a percepção da relação letra-código.

Dentro do contexto de aprendizagem auxiliada por computação móvel, algumas iniciativas de aplicativos móveis voltados à aprendizagem e a prática do Braille podem ser encontrados nas lojas de aplicativos Google Play e AppStore, dos sistemas operacionais Android e iOS. Em uma pesquisa realizada no mês de outubro de 2013 nessas lojas virtuais, os autores desse artigo obtiveram 35 resultados para iPad, 60 resultados para iPhone e 54 resultados para dispositivos Android. Percebe-se que esses aplicativos não foram construídos com o foco no público com deficiência visual, pois carecem de recursos que promovem a acessibilidade. Além disso, tais softwares limitam-se ao estudo da simbologia, e seu uso em práticas pedagógicas em sala de aula

\footnotetext{
${ }^{2}$ http://www.braillevirtual.fe.usp.br/

${ }^{3}$ http://kodbraillebm.ses.usm.my/

${ }^{4}$ Pessoas que possuem visão normal
} 
encontra-se restrito a este fim, não estando integrado às disciplinas de base comum, como, por exemplo, as que trabalham as linguagens, códigos e as suas tecnologias.

Um projeto mais relacionado a este trabalho é o VBGhost [MILNE; BENNETT; LADNER 2013], um jogo móvel educativo para crianças com deficiência visual que ajuda no processo de aprendizado do Braille. O jogo se inicia com o jogador informando uma letra e passando a vez ao próximo jogador, que adiciona uma letra à palavra que está em formação. O objetivo do jogo é evitar ser o jogador que informa a última letra, formando uma palavra completa. Deste modo funciona como um software móvel promotor de inclusão. Esse jogo móvel também utiliza um teclado virtual Braille, entretanto, este não se baseia em um paradigma de múltiplos gestos, o que torna a escrita mais lenta.

\section{Considerações finais e trabalhos futuros}

Na perspectiva da educação inclusiva, a tecnologia é voltada a favorecer a participação do aluno com deficiência nas diversas atividades do cotidiano escolar. Este artigo apresenta os primeiros esforços de desenvolvimento de um conjunto de aplicativos móveis, o GBraille Suite, que visam auxiliar a prática da escrita Braille e sua inserção nas práticas de escrita de Português (e.g., ortografia, sinonímia). Os resultados obtidos apontam para um promissor potencial dessa linha de pesquisa e que as experiências podem ser estendidas a um número maior de participantes. Verificou-se que a lógica de funcionamento do teclado apresenta um pouco de complexidade, pois as pessoas com deficiência visual tendem a pensar o Braille com colunas numeradas, ao invés de linhas. Portanto, necessita-se de um tempo maior dedicado à prática da ferramenta e disponibilizar, nos softwares educativos, suporte para que o usuário possa escolher outros tipos de teclado Braille virtuais. Vale ressaltar, entretanto, que mesmo com essa mudança de paradigma, o tempo de escrita foi bem menor do que no LêBraille.

Como trabalhos futuros, serão desenvolvidos um aplicativo de criação de microcontos (usando SMS) e um audiogame com temática de aventura que utilizará o teclado virtual GBraille como uma das suas mecânicas principais. Finalmente, além do melhoramento e enriquecimento da proposta, resulta ser fundamental um estudo mais sistemático e profundo da avaliação da usabilidade do sistema e do seu impacto nas aplicações feitas com alunos na escola para educação especial parceira do projeto.

\section{Agradecimentos}

Este trabalho é o resultado parcial do projeto GBraille apoiado pelo CNPq (MCT / CNPq 14/2013 - Universal), sob número de concessão 484.255 / 2013-4 e foi apoiado pelo Programa STIC-AmSud-CAPES / CONICYT / MAEE, Projeto KIGB-Knowing and Interacting while Gaming for the Blind, 2014.

\section{Referências}

ARCHAMBAUlT, D.; BURGER, D. TIM (Tactile Interactive Multimedia): Development and adaptation of computer games for young blind children. Proc. ERCIM WG UI4ALL \& i3 Spring Days 2000 Joint workshop, Interactive Learning Environments for Children, Atenas, Grécia, 2000.

AZENKOT, S. et al. Input Finger Detection for Nonvisual Touch Screen Text Entry in Perkinput. GI '12 Proceedings of Graphics Interface 2012, p. 121-129, 2012. 
FAÇANHA, A. R. et al. Touchscreen Mobile Phones Virtual Keyboarding for People with Visual Disabilities. 16th International Conference, HCI International 2014, v. 8512, p. 134-145, 2014.

IDC. Smartphone OS Market Share, Q1 2014. 2014. Disponível em: < http://www.idc.com/prodserv/smartphone-os-market-share.jsp >. Acesso em: 28 jun 2014.

INPRIS. UpSense User Guide. 2012. Disponível em: < http://www.inprisltd.com/media/UpSenseGuide-English.pdf $>$. Acesso em: 16 mai. 2014.

KALRA, N. et al. Iterative Design of A Braille Writing Tutor to Combat Illiteracy. Proceedings of the 2nd IEEE/ACM International Conference on Information and Communication Technologies and Development, 2007.

MILNE, L. R.; BENNETT, C. L.; LADNER, R. E. VBGhost: a braille-based educational smartphone game for children. Proceedings of the 15 th International ACM SIGACCESS Conference on Computers and Accessibility, p. 1-2, 2013.

NICOLAU, H. et al. UbiBraille Designing and Evaluating a Vibrotactile BrailleReading Device. Proceedings of the 15th International ACM SIGACCESS Conference on Computers and Accessibility (ASSETS), 2013.

OHTSUKA, S. et al. Body-Braille System for Disabled People. Proc. of ICCHP 2008, p. 682-685, 2008.

OLIVEIRA, J. et al. BrailleType: Unleashing Braille over Touch Screen Mobile Phones. Proceedings of the INTERACT ' 11, 2011.

PEBAM, P. Swipe Type for the visually impaired. 2011. Disponível em: < http://ptamzz.com/\#!/swipetype >. Acesso em: 16 out. 2014.

PEREIRA, O. R. E.; RODRIGUES, J. J. P. C. Survey and analysis of current mobile learning applications and technologies. ACM Computing Surveys (CSUR), v. 46, n. 2, p. 1-35, 2013.

REYNOLDS, C. R.; VANNEST, K. J.; FLETCHER-JANZEN, E. Braille. Encyclopedia of Special Education: John Wiley \& Sons, Inc. 2013.

SAVI, R. et al. Proposta de um Modelo de Avaliação de Jogos Educacionais. 2010.

SOUTHERN, C. et al. An Evaluation of BrailleTouch: Mobile Touchscreen Text Entry for the Visually Impaired. 14th international conference on Human-computer interaction with mobile devices and services, p. 317-326, 2012.

VIANA, W. et al. Towards the semantic and context-aware management of mobile multimedia. Multimedia Tools and Applications, v. 53, n. 2, p. 391-429, 2011. 